\title{
VISIBLE POINT VECTOR SUMMATIONS FROM HYPERCUBE AND HYPERPYRAMID LATTICES
}

\author{
GEOFFREY B. CAMPBELL \\ Centre for Mathematics and its Applications \\ School of Mathematical Sciences \\ Australian National University \\ Canberra, ACT 0200, Australia
}

(Received February 11, 1997)

\begin{abstract}
.
New identities are given, based on ideas related to visible (from the origin) point vectors. Each result was found from summing on vpv lattices dividing space into radial regions from the origin. This is related to recent work by the author in which new partition type infinite products were derived. Also recently, Baake et al [3] and Mosseri [14] considered the 2-D visible lattice points as part of an optical experiment in which the so-called Optical Fourier Transform was applied. Many of the techniques espoused in Glasser and Zucker [11], and in Ninham et al [15] involving Mellin and Möbius inversions are applicable also to the current paper.
\end{abstract}

KEY WORDS AND PHRASES. Combinatorial identities, Combinatorial number theory, Lattice points in specified regions, Partitions (elementary number theory).

1992 AMS SUBJECT CLASSIFICATION CODES. 05A19, 11B75, 11P21, 11 P81.

\section{INTRODUCTION.}

In recent papers by the author [5-10], new infinite product identities were proven. These were termed visible (from the origin) point vector identities, or simply vpv identities. Each result was found from summing on vpv lattices dividing space into radial regions from the origin. In particular, in Campbell [8] the regions considered for the lattice sums were triangular, cubic, hypercubic, pyramidic, or hyperpyramidic depending on the dimension of the space.

Recently also in Baake et al [3] and Mosseri [14] the visible lattice points were considered as part of an optical experiment in which the so-called Optical Fourier Transform was applied to the 2-D vpv's. 
Apostol [2] gave an excellent introduction to the ideas behind vpv's and calculated their density in space Many of the techniques discussed in Glasser and Zucker [11], and in Ninham et al [15] involving Mellin and Möbius inversions are applicable to vpv identities from the author's papers [5-10] The current paper seeks in part to explore this idea further. It then also adds the perspective that the neatest presentation of these results is in the use of the polylogarithm function $\mathrm{Li}_{m}(z)$ as described in Lewin [13], and given below in ((1.2) In Andrews' book on partitions [1] an historical perspective is given of generating functions for partitions, and an introduction to works on plane partitions and vector partitions. The identities of the author's papers [5-10], especially [7] and within contexts such as those presented in Ninham et al [15], and Glasser and Zucker [11] highlight the possibility of modern application of ideas from this book. Each vpv infinite product turned out to be combinatorially equivalent to a theorem on weighted vector partitions. (see Campbell [6])

2. HYPERCUBE IDENTITIES.

The underlying concept for proof of these vpv identities comes from the

LEMMA 2.1. (see Campbell [5-10]) Consider an infinite region raying out of the origin in any Euclidean vector space. The set of all lattice point vectors from the origin in that region is precisely the set of positive integer multiples of the visible point vectors ( $\left.v p v^{\prime} s\right)$ in that region.

We open our considerations with the

THEOREM 2.2. (see Campbell [7]) If $i=1,2, \ldots n ;(n \neq 1)$ then for each $\left|x_{i}\right|<1$, and $b_{i} \in C$,

$$
\begin{aligned}
& \prod_{\substack{\left(a_{1}, a_{n}\right)=1 \\
a_{1} \ldots \ldots a_{n}=1}}\left(1-x_{1}^{a_{1}} \ldots x_{n}^{a_{n}}\right)^{-a_{1}^{-b_{1}} a_{n} b_{n}} \\
& \quad=\exp \left\{\prod_{i=1}^{n} L i_{b_{1}}\left(x_{i}\right)\right\}, \text { provided } \sum_{i=1}^{n} b_{1}=1 .
\end{aligned}
$$

The differentiated logarithm of this result is quite easy to prove using lemma 2.1 as shown in Campbell [5-10] In this and henceforth we use the terminology for the polylogarithm, (see Lewin [13])

$$
\begin{aligned}
\operatorname{Li}_{m}(z) & =\frac{1}{\Gamma(m)} \int_{0}^{\infty} \frac{z t^{m-1}}{e^{t}-z} d t \\
& =\sum_{k=1}^{\infty} \frac{z^{k}}{k^{m}} \quad \Re m>0,|z| \leq 1,
\end{aligned}
$$

and extend this as was done in the author's [5] to

$$
\mathrm{Li}_{-m}(z)=\sum_{k=1}^{\infty} k^{m} z^{k} \quad \Re m>0,|z| \leq 1 .
$$

We note that when $m$ in (2.3) is a positive integer,

$$
\mathrm{Li}_{-m}(z)=\left.\frac{d^{m}}{d t^{m}}\left(1-e^{t}\right)^{-1}\right|_{t=\log z}
$$

Following easily from theorem 1.2 are the two particular cases, 


$$
\begin{array}{cl}
\prod_{\substack{(a, b)=1 \\
a \geq 0, b \geq 1}}\left(1-y^{a} z^{b}\right)^{1 / b}=(1-z)^{1 /(1-y)}, & |y z|<1,|z|<1, \\
\prod_{\substack{(a, b, c)=1 \\
a, b \geq 0, c z 1}}\left(1-x^{a} y^{b} z^{c}\right)^{1 / c}=(1-z)^{1 /(1-x)(1-y)}, & |x y z|,|x z|,|y z|,|z|<1 .
\end{array}
$$

The combinatorial interpretation of (2.5) may be likened to assigning stepping stones to each lattice point in the first quadrant, and likewise assigning a value for the effort involved in each jump to a successive stone. The "effort" in the case of $(2.5)$ has a value of $(-1)^{(a, b)}\left(\begin{array}{l}-1 / b \\ (a, b)\end{array}\right)$ along the vector $\langle a, b\rangle$ from any existing stepping stone. The person jumping (we assume) will jump to a stone which is always further away from the origin. In combinatorial terms, (2.5) states that

THEOREM 2.3. Let $v=\langle a, b\rangle$ be a vector with integer coordinates such that $a \geq 0$ and $b \geq 1$, and assume that in walking from the origin to $v$ we assign an effort of $(-1)^{(a, b)}\left(\begin{array}{l}-1 / b \\ (a, b)\end{array}\right)$. Then the total effort from performing all possible such jumps from the origin to lattice point $v$ in the first quadrant is given by

$$
\sum_{S} \prod_{a_{1}, b_{1}}(-1)^{(a . b)}\left(\begin{array}{c}
-1 / b \\
(a, b)
\end{array}\right)
$$

where $S$ is the set of unrestricted vector partitions of $v$ with integer coordinates such that $a \geq 0$ and $b \geq 1$, and (2.7) is also equal to the coefficient of $y^{a} z^{b}$ in $(1-z)^{-1 /(1-y)}$

Another way of interpreting formulae such as (2.6) is to take logarithms of both sides and equate coefficients of like powers of $z$ yielding essentially

COROLLARY 2.4. If the variables $x, y$, and $z$ are each non-unity then

$$
\sum_{\substack{d \mid m \\(a, b, c, d)=1}} \frac{m x^{d a} y^{d b} z^{d c}}{\left(1-x^{d a}\right)\left(1-y^{d b}\right)\left(1-z^{d c}\right)}=\frac{x y z}{m^{2}(1-x)(1-y)(1-z)}
$$

In this section we give two theorems as appear in Campbell [7-8]. The first of these is

THEOREM 2.5. (see Campbell [8]) If $i=1,2, \ldots . n ;(n \neq 1)$ then for each $\left|x_{n}\right|$, $\left|x_{n} x_{n-1}\right|, \cdots,\left|x_{n} x_{n-1} \ldots x_{1}\right|<1$, and $b_{i} \in C$,

$$
\begin{aligned}
& \exp \left\{\sum_{k=1} \frac{x_{n}^{k}}{k^{b_{n}}}\right\} \prod_{\substack{\left(a_{1} . \\
a_{1}, a_{n}\right)=1 \\
a_{1}, a_{n-1}<a_{n} \\
a_{n} \rightarrow-1>0}}\left(1-x_{1}^{a_{1}} \ldots x_{n}^{a_{n}}\right)^{-a_{1}{ }^{-b_{1}} a_{n}{ }^{-b_{n}}} \\
&=\exp \left\{\sum_{k=1}^{\infty} \prod_{t=1}^{n-1}\left(\sum_{j=1}^{k-1} \frac{x_{1}^{j}}{j^{b_{t}}}\right) \frac{x_{n}^{k}}{k^{b_{n}}}\right\}, \text { provided } \sum_{t=1}^{n} b_{t}=1
\end{aligned}
$$

The logarithm of this result is quite easy to prove using the technique in Campbell [5-10], by summing on the vpv's in the $n$-space hyperpyramid defined by the inequalities:

$$
x_{1}<x_{n}, \quad x_{2}<x_{n}, \quad \ldots, \quad x_{n-1}<x_{n}
$$


in the first $n$-space hyperquadrant, and applying lemma 2.1. Alternatively, the logarithmic derivative of both sides of (2.9) gives rise to an easily proven identity, again using lemma 2.1 in the process. (see Campbell [9])

The corresponding theorem from Campbell [7] was summed very simply over all lattice point vectors in the first hyperquadrant. The $2-D$ and $3-D$ cases of that are as follows:

COROLLARY 2.6. If $|y z|$ and $|z|<1$, and $s+t=1$, then

$$
\begin{aligned}
\exp \left\{\sum_{k \geq 1} \frac{z^{k}}{k^{t}}\right\} \prod_{\substack{(a, b)=1 \\
a<b \\
a>0, b>1}}\left(1-y^{a} z^{b}\right)^{-a^{-s} b^{-t}} \\
\quad=\exp \left\{\frac{z^{1}}{1^{t}}+\left(1+\frac{y^{1}}{1^{s}}\right) \frac{z^{2}}{2^{t}}+\left(1+\frac{y^{1}}{1^{s}}+\frac{y^{2}}{2^{s}}\right) \frac{z^{3}}{3^{t}}+\ldots\right\} .
\end{aligned}
$$

COROLLARY 2.7. If $|x y z|,|y z|$ and $|z|<1$, and $r+s+t=1$, then

$$
\begin{aligned}
\exp \left\{\sum_{k \geq 1} \frac{z^{k}}{k^{t}}\right\} \prod_{\substack{(a, b, b)=1 \\
a, b<c}}\left(1-x^{a} y^{b} z^{c}\right)^{-a^{-b^{-}-b^{-c^{-t}}}} \\
\quad=\exp \left\{\frac{z^{1}}{1^{t}}+\left(1+\frac{x^{1}}{1^{r}}\right)\left(1+\frac{y^{1}}{1^{s}}\right) \frac{z^{2}}{2^{t}}+\left(1+\frac{x^{1}}{1^{r}}+\frac{x^{2}}{2^{r}}\right)\left(1+\frac{y^{1}}{1^{s}}+\frac{y^{2}}{2^{s}}\right) \frac{z^{3}}{3^{t}}+\ldots\right\}
\end{aligned}
$$

A generalized version of theorem 2.2 is

THEOREM 2.8. If $i=1,2, \ldots n ;(n \neq 1)$ then for each $\left|x_{n}\right|,\left|x_{n} x_{n-1}\right|, \cdots,\left|x_{n} x_{n-1} \ldots x_{1}\right|<1$, and $b_{1} \in C$,

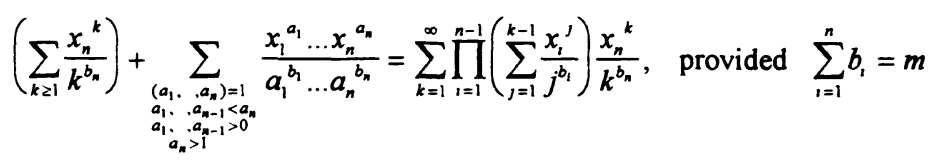

This result is again quite easy to prove by summing on the vpv's in the $n$-space hyperpyramid defined by the inequalities:

$$
x_{1}<x_{n}, \quad x_{2}<x_{n}, \quad \ldots, \quad x_{n-1}<x_{n}
$$

in the first $n$-space hyperquadrant, and applying lemma 2.1 .

The simplest examples are from setting $t=1$ (hence $p, q, r$, and $s=0)$ in (2.11) and (2.12), yielding

$$
\begin{gathered}
\prod_{\substack{(a, b)=1 \\
a b b \\
a \geq 0 . b \geq 1}}\left(1-y^{a} z^{b}\right)^{-b^{-1}}=\left(\frac{1-y z}{1-z}\right)^{\frac{1}{1-y}}, \\
\prod_{\substack{(a, b, c)=1 \\
a, b=c \\
a, b \geq 0.0 \geq 1}}\left(1-x^{a} y^{b} z^{c}\right)^{-c^{-1}}=\left(\frac{(1-x z)(1-y z)}{(1-z)(1-x y z)}\right)^{\frac{1}{(1-x)(1-y)}}
\end{gathered}
$$


$\prod_{\substack{(a, b, c . d)=1 \\ a, b, c<d \\ a . b . c \geq 0 . d \geq 1}}\left(1-w^{a} x^{b} y^{c} z^{d}\right)^{-d^{-1}}=\left(\frac{(1-w z)(1-x z)(1-y z)(1-w x y z)}{(1-z)(1-w x z)(1-w y z)(1-x y z)}\right)^{\frac{1}{(1-w)(1-x)(1-y)}}$

$$
\begin{aligned}
& \prod_{\begin{array}{c}
(a, b, c . d . e)=1 \\
\text { a.b.c.e.e } \\
a . b . c . d \geq 0 . e \geq 1
\end{array}}\left(1-v^{a} w^{b} x^{c} y^{d} z^{e}\right)^{-e^{-1}} \\
& =\left(\frac{(1-v z)(1-w z)(1-x z)(1-y z)(1-v w x z)(1-v w y z)(1-v x y z)(1-w x y z)}{(1-z)(1-v w z)(1-v x z)(1-v y z)(1-w x z)(1-w y z)(1-x y z)(1-v w x y z)}\right)^{\frac{1}{(1-v)(1-w)(1-x)(1-y)}}
\end{aligned}
$$

These results are given in Campbell [6-7] without the full generality of theorem 2.5, and proven in Campbell [9] from another perspective. Particular cases of (2.15) to (2.18) are examined in Campbell [8], where they are shown to have non-trivial simple cases. For example, if each of $v, w, x$, and $y$ are set equal to $z$, then the binomial coefficients appear as exponents in the right sides of (2.15) to (2.18), and (2.18) for example, becomes

$$
\prod_{\substack{(a, b, c, d, e)=1 \\ a, b . c, d<e \\ \text { a.b.c.d }>0, e \geq 1}}\left(1-z^{(a+b+c+d+e)}\right)^{-e^{-1}}=\left(\frac{\left(1-z^{2}\right)^{4}\left(1-z^{4}\right)^{4}}{(1-z)\left(1-z^{3}\right)^{6}\left(1-z^{5}\right)}\right)^{\frac{1}{(1-z)^{4}}} .
$$

This particular case was first given in the author's paper [8]. The right side is easily expanded into a power series in $z$ and the left side when expanded enumerates weighted vpv partitions.

3. A GENERAL HYPERPYRAMID THEOREM.

In Campbell [7] the main theorem of the paper came from summing on all lattice points in the first hyperquadrant. In section 2 this is restricted to hyperpyramid lattices with symmetry in $n-1$ out of the $n$ variables This is evident in viewing (2.9) to (2.13). If we choose to vary the shape of our hyperpyramid the process is identical to the type of multidimensional sum used in the author's [8] to prove theorem 2.2. In [8], which the current paper is sequel to, it becomes clear from this that the general hyperpyramid sums leading to infinite products are included in the

THEOREM 3.1. (see Campbell [8]) For conditions of theorem 2.8, and in addition for $\alpha_{\imath} \in \mathfrak{R}^{+}$we have

$$
\begin{aligned}
& \exp \left\{\sum_{k \geq 1} \frac{x_{n}{ }^{k}}{k^{b_{n}}}\right\} \prod_{\substack{\left(a_{1}, \ldots a_{n}\right)=1 \\
\alpha_{1} a_{1}, a_{n-1} a_{n-1}<a_{n} \\
a_{1}, a_{n-1}>0 \\
a_{n}>1}}\left(1-x_{1}^{a_{1}} \ldots x_{n}^{a_{n}}\right)^{-a_{1}^{-b_{1}} a_{n}{ }^{-b_{n}}} \\
& =\exp \left\{\sum_{k=1}^{\infty} \prod_{i=1}^{n-1}\left(\sum_{j=1}^{F(k)} \frac{x_{i}{ }^{\jmath}}{j^{b_{i}}}\right) \frac{x_{n}{ }^{k}}{k^{b_{n}}}\right\}, \quad \text { provided } \sum_{i=1}^{n} b_{i}=1 \\
& \text { where } F(k)=\left\{\begin{array}{lll}
{\left[\frac{k-1}{\alpha_{t}}\right]} & \text { if } & \alpha_{\imath}>1 . \\
k-1 & \text { if } & \alpha_{\imath}=1 . \\
{\left[\frac{k}{\alpha_{t}}-1\right]} & \text { if } & \alpha_{i}<1 .
\end{array}\right.
\end{aligned}
$$


This theorem counts terms on any possible hyperpyramid lattice in the first hyperquadrant, where the hyperpyramid apex is at the origin. This theorem led to the full range of so called hyperpyramid infinite product identities in [8]. Theorems 2.8 and 3.1 are each cases of the more general

THEOREM 3.2. If $i=1,2, \ldots n ;(n \neq 1)$ then for each $\left|x_{n}\right|,\left|x_{n} x_{n-1}\right|, \cdots,\left|x_{n} x_{n-1} \ldots x_{1}\right|<1$, and $b_{1} \in C$ with $F(k)$ as in (3.2), then provided $\sum_{i=1}^{n} b_{i}=m$,

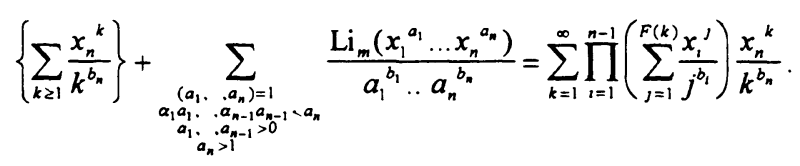

We see that this theorem counts terms on the same hyperpyramid lattice as does theorem 2.2 which is the case where $m=1$.

\section{OUTLINE OF THE PROOF OF THEOREM 3.2, AND SOME EXAMPLES.}

This involves basically the same method given in Campbell [7] and is related also to the method given in [8]. We consider only the case with $n=2$ as it shows the method for the general case, and there is no problem in generalizing the process. We start with the sum valid for the criteria of the theorem

$$
\sum_{b \geq 1} \frac{z^{b}}{b^{t}}+\sum_{\substack{a,<b \\ a>0 . b 22}} \frac{y^{a} z^{b}}{a^{s} b^{t}}=\sum_{k=1}^{\infty}\left(\sum_{j=1}^{F(k)} \frac{y^{j}}{j^{s}}\right) \frac{z^{j}}{k^{t}}
$$

We note by illustration that this is easily seen for $\alpha=1 / 2$ to be

$$
\frac{z^{1}}{1^{t}}+\frac{z^{2}}{2^{1}}\left(1+\frac{y^{1}}{1^{s}}\right)+\frac{z^{3}}{3^{t}}\left(1+\frac{y^{1}}{1^{s}}\right)+\frac{z^{4}}{4^{t}}\left(1+\frac{y^{1}}{1^{5}}\right)+\frac{z^{5}}{5^{t}}\left(1+\frac{y^{1}}{1^{s}}+\frac{y^{2}}{2^{s}}\right)+\cdots
$$

or for $\alpha=1 / 3,(4.1)$ reads as

$$
\frac{z^{1}}{1^{t}}+\frac{z^{2}}{2^{t}}\left(1+\frac{y^{1}}{1^{s}}\right)+\frac{z^{3}}{3^{t}}\left(1+\frac{y^{1}}{1^{s}}\right)+\frac{z^{4}}{4^{t}}\left(1+\frac{y^{1}}{1^{s}}\right)+\frac{z^{5}}{5^{t}}\left(1+\frac{y^{1}}{1^{s}}\right)+\frac{z^{6}}{6^{t}}\left(1+\frac{y^{1}}{1^{s}}+\frac{y^{2}}{2^{s}}\right)+\cdots
$$

Let $m=s+t$. By lemma 2.1, (4.1) also equals

$$
\begin{aligned}
& \left(\sum_{b \geq 1} \frac{z^{b}}{b^{t}}\right)+\sum_{\substack{a,<b \\
(a, b)=1 \\
a \geq 1, b \geq 2}}\left(\frac{\left(y^{a} z^{b}\right)^{1}}{1^{m}}+\frac{\left(y^{a} z^{b}\right)^{2}}{2^{m}}+\frac{\left(y^{a} z^{b}\right)^{3}}{3^{m}}+\cdots\right) \frac{1}{a^{s} b^{t}} \\
& =\mathrm{Li}_{t}(z)+\sum_{\substack{a, x<b \\
(a, b)=1 \\
a \geq 1, b \geq 2}} \frac{\operatorname{Li}_{m}\left(y^{a} z^{b}\right)}{a^{s} b^{t}}
\end{aligned}
$$

so equating (4.1) and (4.4) yields the $n=2$ equivalent of theorem 3.2. The method used here can equally apply to the successive cases $n=3,4,5, \ldots$. Progressing in this manner we see that the method is essentially the same for each dimension. This is due to the symmetry in the non-vertical axes of the lattice. 
The cases presented as corollaries of the theorems so far were given in Campbell [8] although other types of corollaries than infinite products could have been given. That is, the first few examples taken with $m=2$ and successively $n=2,3, \ldots$, are

$$
\begin{gathered}
\left(\sum_{k \geq 1} \frac{z^{k}}{k^{t}}\right)+\sum_{\substack{(a, b)=1 \\
a<b \\
a>0, b>1}} \frac{y^{a} z^{b}}{a^{s} b^{t}}=\sum_{k=1}^{\infty}\left(\sum_{j=1}^{k-1} \frac{y^{j}}{j^{s}}\right) \frac{z^{k}}{k^{k}}, \text { provided } s+t=2 \\
\left(\sum_{k \geq 1} \frac{z^{k}}{k^{t}}\right)+\sum_{\substack{(a, b, c)=1 \\
a, b<c \\
a, b>0, c>1}} \frac{x^{a} y^{b} z^{c}}{a^{\prime} b^{s} c^{t}}=\sum_{k=1}^{\infty}\left(\sum_{j=1}^{k-1} \frac{x^{j}}{j^{r}}\right)\left(\sum_{j=1}^{k-1} \frac{y^{j}}{j^{s}}\right) \frac{z^{k}}{k^{t}}, \text { provided } r+s+t=2 .
\end{gathered}
$$

Both of these appear to be new results

ACKNOWLEDGEMENT. The author thanks Professor George E Andrews for his continued moral support and the Australian National University for their continued association during 1995.

\section{REFERENCES}

1. ANDREWS, G E. The Theory of Partitions, Encyclopedia of Mathematics, Vol. 2, Addison Wesley, 1976

2. APOSTOL, T. Introduction to Analytic Number Theory, Springer-Verlag, New York, 1976.

3. BAAKE, M., GRIMM, U, and WARRINGTON, D. H. Some remarks on the visible points of a lattice, (submitted)

4 BORWEIN, J., and BORWEIN, P. Pi and the AGM., John Wiley and Sons, New York, 1986.

5. CAMPBELL, G. B. A new class of infinite product, and Euler's totient, Int. J. Math. \& Math. Sci., Vol 17, No 4, 1994, 417-422.

6. CAMPBELL, G. B. Dirichlet summations and products over primes, Int. J. Math. \& Math. Sci., Vol 16, No 2, 1993, 359-372.

7 CAMPBELL, G. B. Infinite products over visible lattice points, Int. J. Math. \& Math. Sci., Vol 17, No 4, 1994, 637-654.

8. CAMPBELL, G. B. Infinite products over hyperpyramid lattices, Int. J. Math. \& Math. Sci., (to appear)

9 CAMPBELL, G. B. A closer look at some new identities, Int. J. Math. \& Math. Sci., (to appear)

10 CAMPBELL, G. B. Multiplicative functions over Riemann zeta function products, J. Ramanujan Soc. 7 No. 1, 1992, 52-63.

11. GLASSER, M. L., and ZUCKER, I. J. Lattice Sums, Th. Chem.: Adv. Persp., Vol 5, 1980, $67-$ 139 
12. HARDY, G. H., and WRIGHT, E. M. An Introduction to the Theory of Numbers, Oxford University Press, Clarendon, London, 1971.

13. LEWIN, L. Polylogarithms and Associated Functions, North-Holland, New York, 1981.

14. MOSSERI, $R$ Visible points in a lattice, J. Phys. A25, 1992, L25-29

15. NINHAM, B. W., GLASSER, M. L., HUGHES, B. D., and FRANKEL, N. E. Möbius, Mellin, and Mathematical Physics, Physica A, 186, 1992, 441-481 


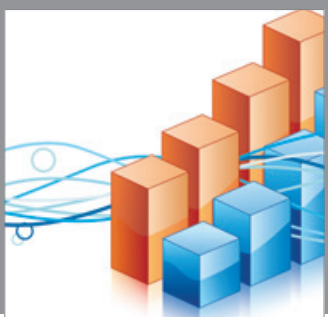

Advances in

Operations Research

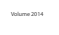

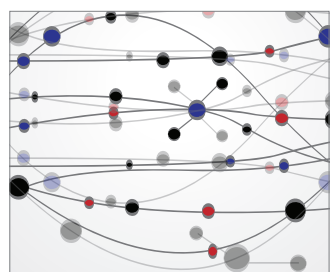

\section{The Scientific} World Journal
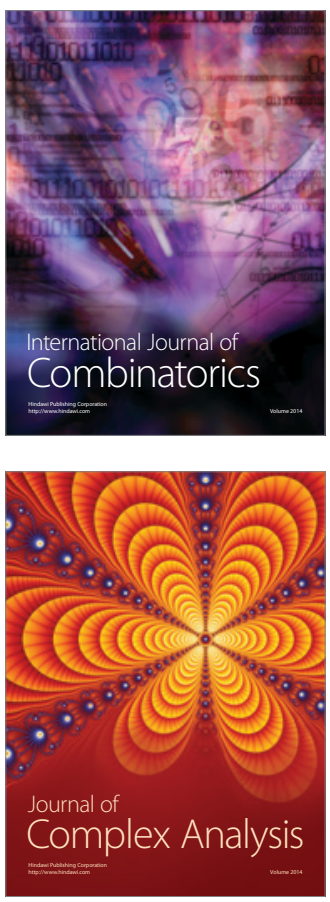

International Journal of

Mathematics and

Mathematical

Sciences
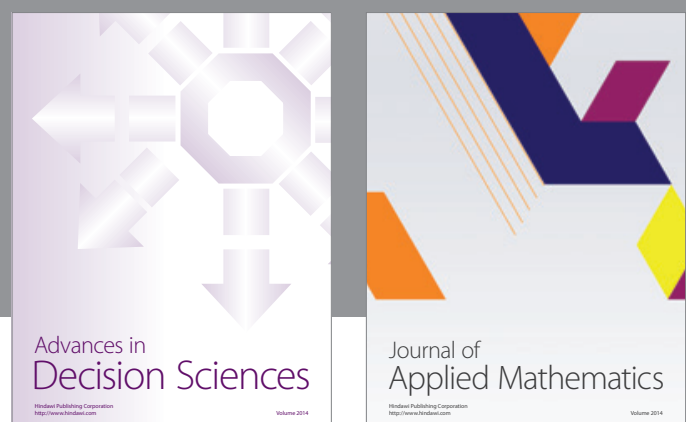

Journal of

Applied Mathematics
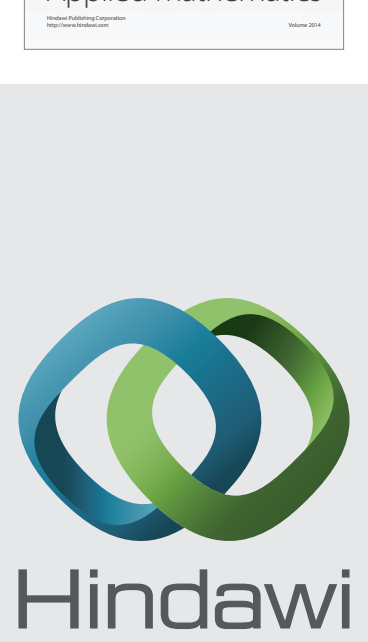

Submit your manuscripts at http://www.hindawi.com
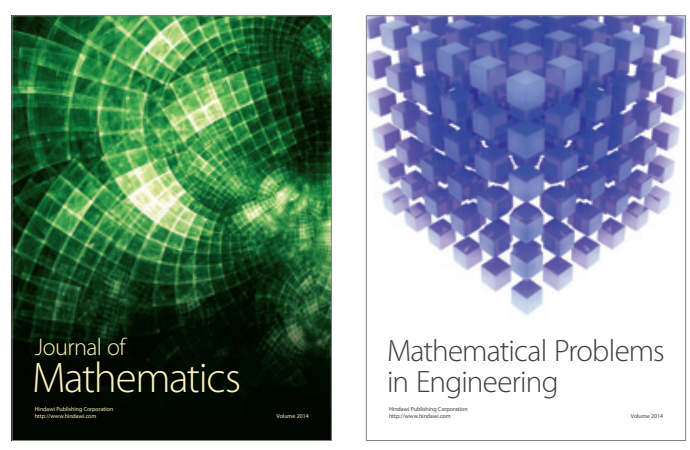

Mathematical Problems in Engineering
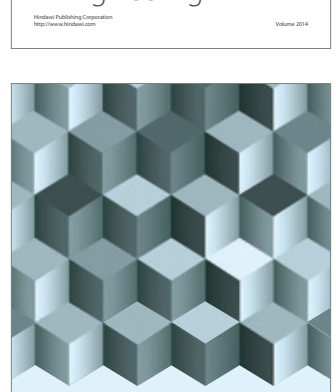

Journal of

Function Spaces
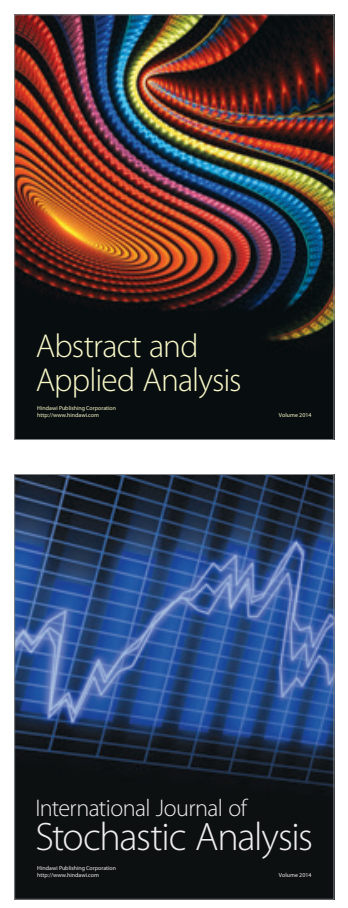

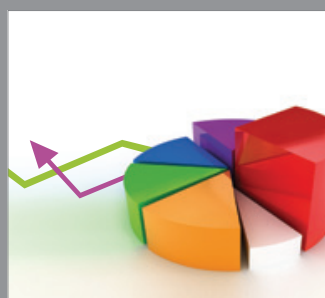

ournal of

Probability and Statistics

Promensencen
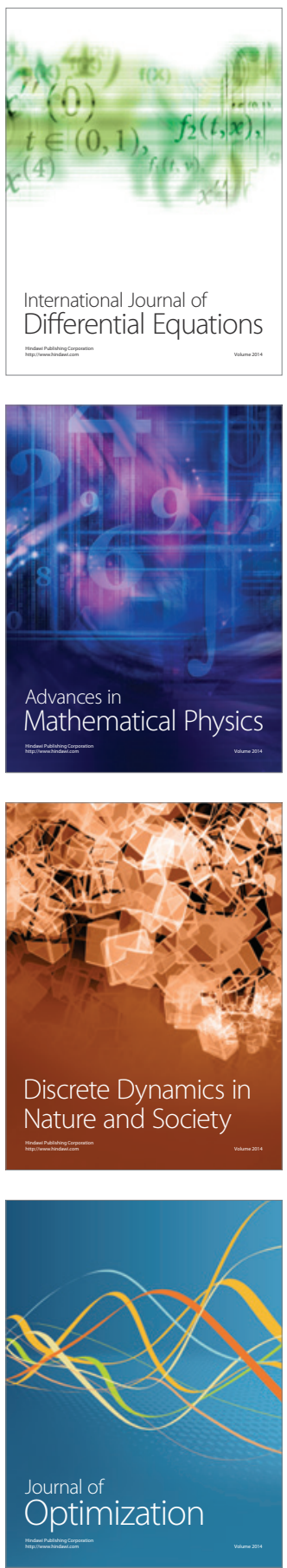